\title{
Unmanned aerial vehicle-aided cooperative regenerative relaying network under various environments
}

\author{
Thanh-Luan Nguyen ${ }^{1}$, Duy-Hung $\mathrm{Ha}^{2}$, Phu Tran Tin ${ }^{3}$, Hien Dinh Cong ${ }^{4}$ \\ ${ }^{1,3}$ Faculty of Electronics Technology, Industrial University of Ho Chi Minh City (IUH), Vietnam \\ ${ }^{2}$ Wireless Communications Research Group, Faculty of Electrical and Electronics Engineering, \\ Ton Duc Thang University, Ho Chi Minh City, Vietnam \\ ${ }^{4}$ Goldwind International Vietnam Co. Ltd., Ho Chi Minh City, Vietnam
}

\begin{tabular}{l}
\hline \hline Article Info \\
\hline Article history: \\
Received Dec 31, 2020 \\
Revised May 6, 2021 \\
Accepted May 17, 2021 \\
\hline
\end{tabular}

Keywords:

Air-to-ground channel

Amplify-and-forward

Energy harvesting

Ground-to-air channel

Unmanned aerial vehicle

\begin{abstract}
This paper studies a cooperative relay network that comprises an unmanned aerial vehicle (UAV) enabling amplify-and-forward (AF) and power splitting (PS) based energy harvesting. The considered system can be constructed in various environments such as suburban, urban, dense urban, and high-rise urban where the air-to-ground channels are model by a mixture of Rayleigh and Nakagami-m fading. Then, outage probability and ergodic capacity are provided under different environment-based parameters. Optimal PS ratios are also provided under normal and high transmit power regimes. Finally, the accuracy of the analytical results is validated through Monte Carlo methods.
\end{abstract}

This is an open access article under the CC BY-SA license.

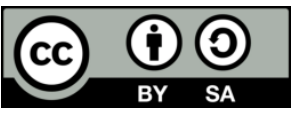

\section{Corresponding Author:}

Duy-Hung Ha

Wireless Communications Research Group

Faculty of Electrical and Electronics Engineering

Ton Duc Thang University

Ho Chi Minh City, Vietnam

Email: haduyhung@tdtu.edu.vn

\section{INTRODUCTION}

Enhancing and prolonging the lifetime of wireless networks under various environment has been a crucial mission for future green communication networks. One promising approach is to deploy unmanned aerial vehicles (UAVs) into practices to meet various exceptional requirements in different environment, e.g., disastrous land site, military in mountainous areas, and traffic control in dense urban [1]-[6]. Among these applications, ground equipments served by UAVs can either experience line-of-sight (LoS) or non-line-ofsight $(\mathrm{nLoS})$ links. Therefore, UAV can play a major role in enhancing throughput as well as greatly improve reliability [7], [8].

Exploiting UAV as a relay in cooperative communication scenarios has attracted many researchers and there are still ongoing works on such topics. In fact, UAV possess flexible deployment characteristics and thus various systematic parameters such as average throughput, and spectral efficiency, can be greatly enhanced by optimizing UAV placement in 3D Euclidean space, i.e., trajectory optimization [9]-[14], deploying multiple-UAV to assist ground equipment [13], [14], power control [15], [16] and resource allocation [17]. Power splitting-based energy harvesting (PSEH) has been viewed as a future technology that can exploit a portion of radio frequency (RF) signal to recharge the battery in energy-constrained equipment [18]. Up to now, there are many works on the performance analysis of UAV-aided cooperative relay network with PSEH. However, the performance under different communication environments exploiting A2G/G2A channel characteristic is still not reported in the literature. 
Motivated by the aforesaid works, this paper examines the performance of UAV-aided cooperative regenerative relaying network under various environments, such as suburban, urban, dense-urban, and highrise urban. In addition, PSEH technology is employed to opportunistically prolong the flight time of the UAV. Particularly, the UAV harvests energy from the ground transmitter (GT) and then consumes the harvested energy to forward information to the ground receiver (GR). Amplify-and-forward (AF) relaying scheme is employed at the UAV to assist the information exchange from GT to the desired GR. Moreover, air-to-ground (A2G) and ground-to-air (G2A) channels are modeled with respect to LoS and nLoS conditions. Then, a closed-form expression for the outage probability and the average throughput under various environments are then provided. Optimal parameters of the PSEH receiver are also studied for deployment insights. The main contributions of this research can be focused on as the following:

- We propose an UAV-assisted relay system under various environments where the relay node jointly uses AF relaying and PSEH protocol to help terrestrial nodes.

- The performance of the considering system is examined over various environments, in which novel analytical results for the outage probability and ergodic capacity are given.

- Then, the results are validated via Monte Carlo method.

\section{RESEARCH METHOD}

Consider an UAV-assisted relay system in which the ground transmitter (GT) wishes to communicate with the ground receiver (GR). Assuming that the direct GT-to-GR link is unavailable due to multiple blockages and heavy shadowing and thus a PSEH-enabled UAV (V) using AF relaying protocol is deployed to assist the terrestrial communication. The harvested energy from the ground transmitter is exploited to forward the information to the ground receiver. We assume the block fading model, in which all channels are invariant during a specific time block $T$ but then varies independently from other blocks. All nodes are assumed to be equipped with a single half-duplex antenna. The A2G and G2A channels, i.e., the UAV-to-GR (V2R) and GT-to-UAV (T2V) channels, are modeled via LoS and nLoS small-scale and largescale propagation.

\subsection{The A2G/G2A channel model}

In this paper, the A2G and G2A channel model proposed in [19] is adopted. Particularly, let $h_{X V}$ be the channel coefficient between node $\mathcal{X} \in\{T, R\}$ and the UAV, thus

$$
h_{X V}=\left\{\begin{array}{l}
g_{X V} \sqrt{a_{X V} d_{X V}^{-\varepsilon}}, \text { for LoS links } \\
g_{X V} \sqrt{\bar{a}_{X V} d_{X V}^{-\varepsilon}}, \text { for LoSn links }
\end{array}\right.
$$

in which $\alpha_{X V}$ and $\bar{a}_{X V}$ are the attenuation coefficients under LoS and nLoS condition, respectively, $d_{X V}$ is the distance between $\mathrm{X}$ and the UAV and $\varepsilon$ is the path loss exponent. Accordingly, the probability of LoS connection can be modeled by Bernoulli distribution with parameter [19] $\rho_{X V}=[1+\psi \exp (-\omega(\theta-\psi))]^{-1}$, in which $\psi$ and $\omega$ are the environmental parameters, $\theta=180 \arctan \left(h_{V} r_{X V}^{-1} / \pi\right)$ denotes the elevation angle of the UAV with $h_{V}$ being the distance between the UAV and the ground, and $r_{X V}$ is the distance between node $\mathrm{X}$ to the projection of UAV in the ground. In addition, the probability of nLoS link also follows Bernoulli distribution with parameter $\bar{\rho}_{X V}=1-\rho_{X V}$. Further, the small-scale propagation of the A2G and G2A link is modeled by Nakgami- $m$ fading for the LoS links and Rayleigh fading for the nLoS links. Particularly, the PDF of the channel power gains $\left|g_{X V}\right|^{2}$ and $\left|\bar{g}_{X V}\right|^{2}$ follow Gamma and exponential distribution, respectively. Hence, the PDFs of $\left|g_{X V}\right|^{2}$ and $\left|\bar{g}_{X V}\right|^{2}$ are given by [20],

$$
\begin{aligned}
& f_{X V^{2}}(x)=\frac{1}{\left(m_{X V}-1\right) !}\left(\frac{m_{X V}}{\Omega_{X V}}\right)^{m_{X V}} x^{m_{X V^{-1}}} e^{-\frac{m_{X V}}{\Omega_{X V}}}, x>0 \\
& f_{X V^{2}}(x)=\frac{1}{\Omega_{X V}} e^{-\frac{x}{\Omega_{X V}}}, x>0
\end{aligned}
$$

respectively, where $\Omega_{X V}$ specifies the average channel power gain. In addition, the corresponding CDFs can be expressed as (4), (5);

$$
f_{X V^{2}}(x)=1-e^{-\frac{x m_{X V}}{\Omega_{X V}} \sum_{m=0}^{m} m^{-1} \frac{x^{m}}{m !}\left(\frac{m_{X V}}{\Omega_{X V}}\right)^{m}}, x>0
$$




$$
f_{X V^{2}}(x)=1-e^{-\frac{x m_{X V}}{\Omega_{X V}}}, x>0
$$

\subsection{The A2G/G2A channel model}

During the first time slot, the GT broadcasts unit energy information signal $S_{T}$. Therefore, the received signal at the information receiver in the UAV can be formulated as (6),

$$
y_{V}=\sqrt{(1-\beta)} P_{T} h_{T V} s_{T}+n_{V}
$$

in which $n_{V} \sim \mathcal{C} \mathcal{N}\left(0, \sigma^{2}\right)$ specifies the additive white Gaussian noise (AWGN) with zero mean and variance $\sigma^{2}, P_{T}$ denotes the transmit power and $\beta \epsilon(0,1)$ denotes the power-splitting ratio.

At the UAV, a portion of the received power is exploited for energy harvesting. Assuming that the power harvested from the AWGN is negligible, the harvested power due to PS-based EH scheme can then be given by [21]-[24] $P_{V}=\eta \beta P_{T}\left|h_{T V}\right|^{2}$ in which the coefficients $\eta \epsilon(0,1)$ presents the DC-to-RF conversion efficiency.

Subsequently, the UAV amplifies the received signal $y_{V}$ with the variable gain factor $G_{V}=$ $\sqrt{\frac{P_{V}}{(1-\beta) P_{T}\left|h_{T V}\right|^{2}+\sigma^{2}}}$. Accordingly, the amplified signal received at GR can then be formulated as (7), (8):

$$
\begin{aligned}
& y_{R}=G_{V} h_{R V} y_{V}+n_{R} \\
& =\underbrace{G_{V} h_{R V} \sqrt{(1-\beta)} P_{T} h_{T V} s_{T}}_{\text {amlified inforamation signal }}+\underbrace{G_{V} h_{R V} n_{V}}_{\text {amplified } A W G N}+\underbrace{n_{R}}_{A W G N}
\end{aligned}
$$

in which $n_{V} \sim \mathcal{C} \mathcal{N}\left(0, \sigma^{2}\right)$ denotes the AWGN at the GR. and (10);

Subsequently, the received signal-to-interference-plus-noise ratio (SINR) can then be given by (9)

$$
\begin{aligned}
& \gamma_{e 2 e}=\frac{\left|G_{V}\right|^{2}\left|h_{T V}\right|^{2}(1-\beta) P_{T}\left|h_{T V}\right|^{2}}{\left|G_{V}\right|^{2}\left|h_{T V}\right|^{2} \sigma^{2}} \\
& =\frac{\beta(1-\beta) \gamma_{R V}\left(\bar{\gamma}_{T}+\gamma_{T V}\right)^{2}}{\beta \gamma_{R V} \bar{\gamma}_{T} \gamma_{T V}+(1-\beta) \bar{\gamma}_{T} \gamma_{T V}+1}
\end{aligned}
$$

in which $\gamma_{T V} \triangleq\left|h_{T V}\right|^{2}, \gamma_{R V} \triangleq \eta\left|h_{R V}\right|^{2}$ and $\bar{\gamma}_{T} \triangleq P_{T} / \sigma^{2}$. In addition, at the high transmit signal-to-noise ratio (SNR) regime $\left(\bar{\gamma}_{T} \rightarrow \infty\right)$, thus $G_{T} \rightarrow \sqrt{\eta \beta /(1-\beta)}$. Hence, the received end-to-end (e2e) can then be upper bounded as (11)

$$
=\gamma_{e 2 e} \leq \frac{\gamma_{R V} \bar{\gamma}_{T} \gamma_{T V}}{\frac{\gamma_{R V}}{1-\beta}+\frac{1}{\beta}} \triangleq \gamma_{e 2 e}^{u p p}
$$

\subsection{Performance evaluation}

In this subsection, we study the performance of the proposed system. Specifically, bounds for the outage probability and Ergodic capacity are introduced and later expressed in exact analytical-forms. The tightness of the proposed bound will be discussed in section 3 .

\subsubsection{Outage probability}

The outage probability (OP) is defined as the probability the e2e SINR falls below a predefined threshold. In other words, the OP at the ground receiver can be formulated as (12), (13);

$$
\begin{aligned}
& P_{\text {out }}=\operatorname{Pr}\left\{\gamma_{e 2 e}<\gamma_{t h}\right\} \\
& \leq \operatorname{Pr}\left\{\gamma_{e 2 e}^{\text {upp }}<\gamma_{\text {th }}\right\} \triangleq P_{\text {out }}^{\text {low }},
\end{aligned}
$$

where $P_{\text {out }}^{\text {low }}$ specifies the lower bounded e2e OP. From (13), it is depicted that $P_{\text {out }}^{\text {low }}=\widehat{F_{\text {e2e }}}\left(\gamma_{\text {th }}\right)$, where $\widehat{F_{e 2 e}}(x)$ specifies the CDF of the upper bounded e2e SINR. From (11) and (13), the CDF $\widehat{F_{e 2 e}}(x)$ can then be rewritten as (14); 


$$
\begin{aligned}
& \widehat{F_{e 2 e}}(x)=\operatorname{Pr}\left\{\bar{\gamma}_{T} \gamma_{T V}<\frac{\gamma}{1-\beta}+\frac{\gamma}{\beta} \frac{1}{\gamma_{R V}}\right\} \\
& =\int_{0}^{\infty} F_{\gamma_{T V}}\left(\frac{\gamma}{1-\beta}+\frac{\gamma}{\beta_{x}}\right) f_{\gamma_{R V}}(x) d x
\end{aligned}
$$

respectively, in which $\lambda_{T V} \triangleq \Omega_{T V} d_{T V}^{-\varepsilon} \alpha_{T V}, \bar{\lambda}_{T V} \triangleq \Omega_{T V} d_{T V}^{-\varepsilon} \bar{\alpha}_{T V}, \lambda_{R V} \triangleq \eta \Omega_{R V} d_{R V}^{-\varepsilon} \alpha_{R V}$ and $\bar{\lambda}_{R V} \triangleq \eta \Omega_{R V} d_{R V}^{-\varepsilon} \bar{\alpha}_{R V}$. By calculation, the upper-bounded e2e CDF can be given in closed-form as (16),

$$
F_{\mathrm{e} 2 \mathrm{e}}(\gamma)=1-\rho_{\mathrm{TV}} \rho_{\mathrm{RV}} G_{1}(\gamma)-\rho_{\mathrm{TV}} \bar{\rho}_{\mathrm{RV}} G_{2}(\gamma)-\bar{\rho}_{\mathrm{TV}} \rho_{\mathrm{RV}} G_{3}(\gamma)-\bar{\rho}_{\mathrm{TV}} \bar{\rho}_{\mathrm{RV}} G_{4}(\gamma)
$$

in which $G_{1}(\gamma) \triangleq G\left(m_{\mathrm{TV}}, m_{\mathrm{RV}}, \lambda_{\mathrm{TV}}, \lambda_{\mathrm{RV}} ; \gamma\right), G_{2}(\gamma) \triangleq G\left(m_{\mathrm{TV}}, 1, \lambda_{\mathrm{TV}}, \bar{\lambda}_{\mathrm{RV}} ; \gamma\right), \quad G_{3}(\gamma) \triangleq G\left(1, m_{\mathrm{RV}}, \bar{\lambda}_{\mathrm{TV}}, \lambda_{\mathrm{RV}} ; \gamma\right) \quad$ and $G_{4}(\gamma) \triangleq G\left(1,1, \bar{\lambda}_{\mathrm{TV}}, \bar{\lambda}_{\mathrm{RV}} ; \gamma\right)$ where the self-defined function $G(\cdot)$ is presented by (17);

$$
\begin{gathered}
G\left(m_{1}, m_{2}, \lambda_{1}, \lambda_{2} ; \gamma\right) \triangleq \frac{2}{\left(m_{2}-1\right) !} \exp \left(-\frac{m_{1}}{\lambda_{1}} \frac{1}{1-\beta} \frac{\gamma}{\bar{\gamma}_{\mathrm{T}}}\right)^{m_{\mathrm{TV}}-1} \sum_{m=0}^{1} \frac{1}{m !}\left(\frac{m_{1}}{\lambda_{1}} \frac{1}{1-\beta} \frac{\gamma}{\bar{\gamma}_{\mathrm{T}}}\right)^{m} \\
\times \sum_{k=0}^{m}\left(\begin{array}{l}
m \\
k
\end{array}\right)\left(\frac{1-\beta}{\beta} \frac{m_{2}}{\lambda_{2}}\right)^{k}\left(\frac{m_{1} m_{2}}{\lambda_{1} \lambda_{2}} \frac{\gamma}{\beta} \bar{\gamma}_{\mathrm{T}}\right)^{\frac{m_{2}-k}{2}} K_{m_{2}-k}\left(2 \sqrt{\frac{m_{1} m_{2}}{\lambda_{1} \lambda_{2}} \frac{\gamma}{\beta \bar{\gamma}_{\mathrm{T}}}}\right),
\end{gathered}
$$

in which $K_{v}(x)$ denotes the $v$-th order modified Bessel function of the second kind.

Proof: In order to derive (15) in closed-form, the CDF $F_{\gamma_{T v}}(x)$ and the $f_{\gamma_{R V}}(x)$ are required. Recalling that the probability of LoS and nLoS paths are modeled by Bernoulli distribution, thus

$$
\begin{aligned}
& F_{\gamma_{\mathrm{XV}}}(x)=\rho_{\mathrm{XV}} \operatorname{Pr}\left\{\left|g_{\mathrm{XV}}\right|^{2} \lambda_{\mathrm{XV}}<x\right\}+\bar{\rho}_{\mathrm{XV}} \operatorname{Pr}\left\{\left|\bar{g}_{\mathrm{XV}}\right|^{2} \bar{\lambda}_{\mathrm{XV}}<x\right\} \\
& =\rho_{\mathrm{XV}} F_{\left|g_{\mathrm{xV}}\right|^{2}}\left(x / \lambda_{\mathrm{XV}}\right)+\bar{\rho}_{\mathrm{XV}} F_{\left|\bar{g}_{\mathrm{XV}}\right|^{2}}\left(x / \bar{\lambda}_{\mathrm{XV}}\right), \\
& f_{\gamma_{\mathrm{XV}}}(x)=\left(\rho_{\mathrm{XV}} / \lambda_{\mathrm{XV}}\right) f_{g_{\mathrm{Xv}}}\left(x / \lambda_{\mathrm{XV}}\right)+\left(\bar{\rho}_{\mathrm{XV}} / \bar{\lambda}_{\mathrm{XV}}\right) f_{\bar{g}_{\mathrm{Xv}}}\left(x / \bar{\lambda}_{\mathrm{XV}}\right),
\end{aligned}
$$

in which $X \epsilon\{T, V\}$. By adopting the above results, we can rewrite (15) as (20);

$$
\begin{aligned}
& \widehat{F_{e 2 e}}(\gamma)=1-\rho_{T V} \rho_{R V} \underbrace{\int_{0}^{\infty}\left[1-F_{\left|g_{T V}\right|^{2}}\left(\frac{\gamma}{(1-\beta) \lambda_{T V}}+\frac{\gamma}{\beta \lambda_{T V}} \frac{1}{x}\right)\right]} \frac{1}{\lambda_{R V}} F_{\left|g_{R V}\right|^{2}}\left(\frac{x}{\lambda_{R V}}\right) d x \\
& -\rho_{T V} \bar{\rho}_{R V} \underbrace{\int_{0}^{\infty}\left[1-F_{\left|g_{T V}\right|^{2}}\left(\frac{\gamma}{(1-\beta) \lambda_{T V}}+\frac{\gamma}{\beta \lambda_{T V}} \frac{1}{x}\right)\right]} \frac{1}{\lambda_{R V}} F_{\left|\bar{g}_{R V}\right|^{2}}\left(\frac{x}{\lambda_{R V}}\right) d x \\
& -\bar{\rho}_{T V} \rho_{R V} \underbrace{\int_{0}^{\infty}\left[1-F_{\left|\bar{g}_{T V}\right|^{2}}\left(\frac{\gamma}{(1-\beta) \lambda_{T V}}+\frac{\gamma}{\beta \lambda_{T V}} \frac{1}{x}\right)\right]} \frac{1}{\lambda_{R V}} F_{\left|g_{R V}\right|^{2}}\left(\frac{x}{\lambda_{R V}}\right) d x \\
& -\bar{\rho}_{T V} \bar{\rho}_{R V} \underbrace{\int_{0}^{\infty}\left[1-F_{\left|\bar{g}_{T V}\right|^{2}}\left(\frac{\gamma}{(1-\beta) \lambda_{T V}}+\frac{\gamma}{\beta \lambda_{T V}} \frac{1}{x}\right)\right]}_{G_{4}(\gamma)} \frac{1}{\lambda_{R V}} F_{\left|\bar{g}_{R V}\right|^{2}}\left(\frac{x}{\lambda_{R V}}\right) d x
\end{aligned}
$$

In (20), analysis for the above integrals is analogous to one another. Particularly, the first integral can be given as (21);

$$
\begin{aligned}
& G_{1}(\gamma) \triangleq \sum_{m=0}^{m_{T V}-1} \frac{1}{m !}\left(m_{T V}\right)^{m} \exp \left(-m_{T V} \frac{\gamma}{(1-\beta) \lambda_{T V}}\right) \frac{1}{\lambda_{R V}} \frac{\left(m_{R V}\right)^{m_{R V}}}{\left(m_{R V}-1\right) !} \\
& \times \int_{0}^{\infty} x^{m_{T V}-1}\left(\frac{\gamma}{(1-\beta) \lambda_{T V}}+\frac{\gamma}{\beta \lambda_{T V}} \frac{1}{x}\right)^{m} \exp \left(-m_{T V} \frac{\gamma}{\beta \lambda_{T V}} \frac{1}{x}--m_{R V} \frac{\gamma}{\lambda_{R V}}\right) d x
\end{aligned}
$$

By applying binomial theorem, i.e., $(x+y)^{m}=\sum_{k=0}^{m}\left(\begin{array}{c}m \\ k\end{array}\right) x^{m-k} y^{k}$ and then adopting [25], (3.471.9), we then further express $G_{1}(\gamma)$ as (25), 


$$
\begin{aligned}
& G_{1}(\gamma) \triangleq \frac{2}{\left(m_{R v}-1\right)} \exp \left(-\frac{m_{T v}}{\lambda_{T v}} \frac{1}{1-\beta} \frac{\gamma}{\bar{\gamma}_{T}}\right) \sum_{m=0}^{m_{R v}-1} \frac{1}{m !}\left(\frac{m_{T v}}{\lambda_{T v}} \frac{1}{1-\beta} \frac{\gamma}{\bar{\gamma}_{T}}\right)^{m} \\
& x \sum_{k=0}^{m}\left(\begin{array}{c}
m \\
k
\end{array}\right)\left(\frac{1-\beta}{\beta} \frac{m_{R V}}{\lambda_{R V}}\right)^{k}\left(\frac{m_{T v} m_{R V}}{\lambda_{T V} \lambda_{R V}} \frac{\gamma}{\beta \bar{\gamma}_{T}}\right)^{\frac{m_{2}-k}{2}} K_{m_{2}-k}\left(2\left(\sqrt{\frac{m_{T v} m_{R V}}{\lambda_{T V} \lambda_{R V}} \frac{\gamma}{\beta \bar{\gamma}_{T}}}\right)\right.
\end{aligned}
$$

In addition, the integrals $G_{2}(\gamma), G_{3}(\gamma)$ and $G_{4}(\gamma)$ can be analogously expressed via the above equation, which can then be expressed via the defined function $G\left(m_{1}, m_{2}, \lambda_{1}, \lambda_{2} ; \gamma\right)$. Subsequently, we then obtain the desired result.

\subsubsection{Ergodic capacity}

Considering fast fading scenarios and the e2e performance is dominated by the channel condition, thus a more suitable performance metrics is Ergodic capacity which can capture the dynamic variant characteristics. Accordingly, the Ergodic capacity is defined via the received e2e SINR observed during the effective transmission time block, or in other words,

$$
\begin{aligned}
& C_{e r g}=\frac{1}{2} E\left[\log _{2}\left(1+\gamma_{e 2 e}\right)\right] \\
& \leq \frac{1}{2} E\left[\log _{2}\left(1+\gamma_{e 2 e}^{u p p}\right)\right] \triangleq \widehat{C_{e r g}}
\end{aligned}
$$

Consequently, the upper bounded Ergodic capacity, $\widehat{C_{\text {erg }}}$, can then be given by [26],

$$
\widehat{C_{e r g}}=\frac{1}{2 \operatorname{In} 2} \int_{0}^{\sim} \frac{1}{1+\gamma}\left[1-\widehat{F_{e 2 e}}(\gamma)\right] d_{\gamma}
$$

\subsection{Optimal power splitting ratio}

In this subsection, the value of $\beta^{*}$ optimizing the e2e performance is formulated. First, we formulate the optimization problem as (26), (27);

$$
\begin{aligned}
& \beta^{*}=\begin{array}{l}
\max \\
0<\beta<1
\end{array} \gamma_{e 2 e} \\
& =\min _{0<\beta<1} \underbrace{\frac{\gamma_{R V} \bar{\gamma}_{T} \gamma_{T V}}{1-\beta}+\frac{\bar{\gamma}_{T} \gamma_{T V}}{\beta}+\frac{1}{\beta(1-\beta)}}_{f(\beta)}
\end{aligned}
$$

Since $\frac{\gamma_{R V} \bar{\gamma}_{T} \gamma_{T V}}{1-\beta}, \frac{\bar{\gamma}_{T} \gamma_{T V}}{\beta}$, and $\frac{1}{(1-\beta)}$ are convex over the domain $\beta \epsilon(0,1)$, their sum is also convex. Hence, there exists a unique $\beta^{*} \epsilon(0,1)$, minimizing the objective function $f(\beta)$, obtained via the solution of the equality $f^{\prime}(\beta)=0$. Accordingly, $\beta^{*}$ can be obtained as $(28)$;

$$
\beta^{*}=\frac{\sqrt{\bar{\gamma}_{T} \gamma_{T V}+1}}{\sqrt{\bar{\gamma}_{T} \gamma_{T V}+1}+\sqrt{\gamma_{R V} \bar{\gamma}_{T} \gamma_{T V+1}}}
$$

\section{RESULTS AND DISCUSSION}

In this subsection, Monte Carlo simulations are carried out to validate the analysis in previous sections. Various systematic insights are also presented for practical deployment purposes. For simplicity, let us assume that $\alpha_{T V}=\alpha_{R v} \triangleq \alpha$ and $\bar{\alpha}_{T V}=\bar{\alpha}_{R V} \triangleq \bar{\alpha}$. In addition, the environmental parameters adopted in the Figures 1 and 2 are provided in Table 1 .

Table 1. Environmental parameters

\begin{tabular}{ccccc}
\hline & $\psi$ & $\omega$ & $\alpha$ & $\bar{\alpha}$ \\
\hline Sub-Urban & 4.88 & 0.43 & 0.1 & 21 \\
Urban & 9.61 & 0.16 & 1 & 20 \\
Dense Urban & 12.08 & 0.11 & 1.6 & 23 \\
High-Rise Urban & 27.33 & 0.08 & 2.3 & 34 \\
\hline
\end{tabular}


Other simulation parameters are provided as follows. The path loss exponent for the A2G and G2A links are $\varepsilon=2$, the noise power density is $-144 \mathrm{dBm} / \mathrm{Hz}$ and the bandwidth is $10 \mathrm{MHz}$. Further, the shape factors are $m_{T V}=3$ and $m_{R V}=2$, the distances are $h_{V}=2(m), d_{T V}=2(m)$ and $d_{R V}=2(m)$. Finally, the optimal curves utilize $\beta^{*}$ in (28) whereas other curves use $\beta=0.8$ and $\eta=1$.

Figures 1 and 2 show the e2e outage probability and the Ergodic capacity versus the transmit power of the GT under different environmental parameters in Table 1, respectively. It is observed the simulation result in (12), (18) is tightly matched with the upper-bounded result using (16) which shows the effectiveness of the proposed analytical bound. In addition, Figures 1 and 2 show that as the transmit power at the GT increases, both e2e OP and Ergodic capacity can be effectively improved. Under various environments, the system operates badly in the sub-urban region whereas best in high-rise urban areas.

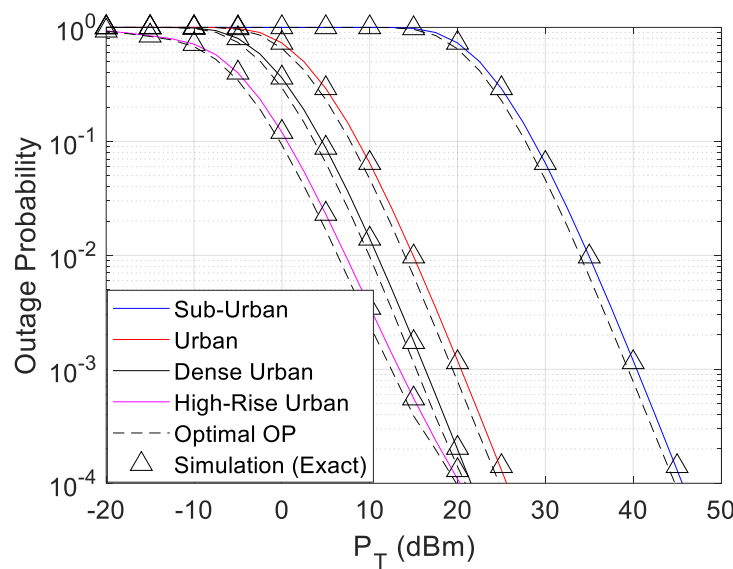

Figure 1. Outage probability versus the transmit power, $P_{T}(\mathrm{dBm})$, under various environments

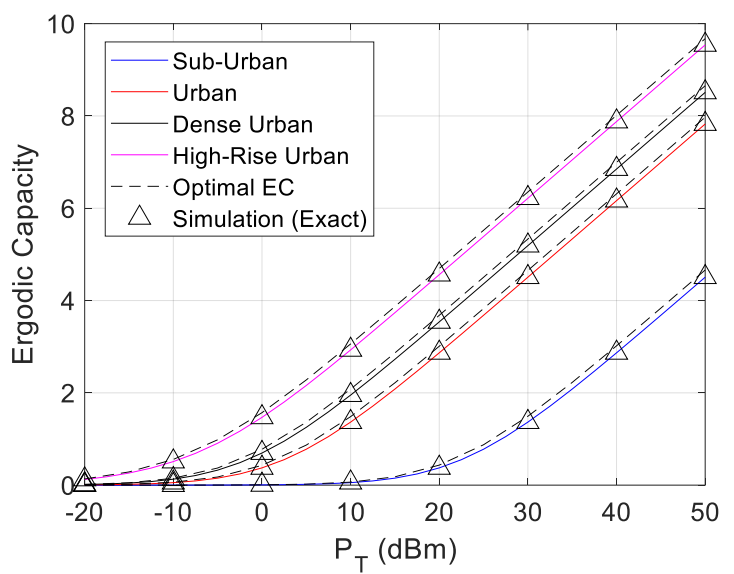

Figure 2. Ergodic capacity versus the transmit power, $P_{T}(\mathrm{dBm})$, under various environments

\section{CONCLUSION}

In this paper, a unified UAV-aided regenerative relay network is proposed which jointly utilizes PSEH and AF protocol to assist the transmission of terrestrial nodes over various environmental settings. Exact expressions of the outage probability and Ergodic capacity in the high transmit power regime are provided. In addition, optimal operational parameters of the energy harvester in the UAV are examined in the normal/high transmit regime. All results are verified by adopting numerical methods and Monte Carlo simulation which shows the effectiveness of the proposed upper bound. The results show that high-rise urban is the most suitable environment to implement UAV as a relay whereas sub-urban is the most incompatible.

\section{ACKNOWLEDGEMENTS}

This research is supported by Industrial University of Ho Chi Minh City (IUH) under grant number 72/HD-DHCN

\section{REFERENCES}

[1] Y. Zeng, R. Zhang, and T. J. Lim, "Throughput maximization for UAV-enabled mobile relaying systems," IEEE Transactions on Communications, vol. 64, no. 12, pp. 4983-4996, 2016, doi: 10.1109/TCOMM.2016.2611512.

[2] F. Jiang and A. L. Swindlehurst, "Optimization of UAV heading for the ground-to-air uplink," IEEE Journal on Selected Areas in Communications, vol. 30, no. 5, pp. 993-1005, 2012, doi: 10.1109/JSAC.2012.120614.

[3] I. Bor-Yaliniz and H. Yanikomeroglu, "The new frontier in RAN heterogeneity: Multi-tier drone-cells," IEEE Communications Magazine, vol. 54, no. 11, pp. 48-55, 2016, doi: 10.1109/MCOM.2016.1600178CM.

[4] H. Menouar, I. Guvenc, K. Akkaya, A. S. Uluagac, A. Kadri, and A. Tuncer, "UAV-enabled intelligent transportation systems for the smart city: Applications and challenges," IEEE Communications Magazine, vol. 55, no. 3, pp. 22-28, 2017, doi: 10.1109/MCOM.2017.1600238CM.

[5] M. Mozaffari, W. Saad, M. Bennis, and M. Debbah, "Mobile unmanned aerial vehicles (UAVs) for energy-efficient internet of things communications," IEEE Transactions on Wireless Communications, vol. 16, no. 11, pp. 7574-7589, 2017, doi: 10.1109/TWC.2017.2751045. 
[6] G. Akhshirsh, N. Al-Salihi, and O. H. Hamid, "A cost-effective GPS-aided autonomous guided vehicle for global path planning," Bulletin of Electrical Engineering and Informatics (BEEI), vol. 10, no. 2, pp. 650-657, 2021, doi: 10.11591/eei.v10i2.2734.

[7] A. Merwaday, A. Tuncer, A. Kumbhar, and I. Guvenc, "Improved throughput coverage in natural disasters: Unmanned aerial base stations for public-safety communications," IEEE Vehicular Technology Magazine, vol. 11, no. 4, pp. 53-60, 2016, doi: 10.1109/MVT.2016.2589970.

[8] Y. Zeng, R. Zhang, and T. J. Lim, "Wireless communications with unmanned aerial vehicles: opportunities and challenges," IEEE Communications Magazine, vol. 54, no. 5, pp. 36-42, 2016, doi: 10.1109/MCOM.2016.7470933.

[9] Y. Zeng, X. Xu, and R. Zhang, "Trajectory design for completion time minimization in UAV-enabled multicasting," IEEE Transactions on Wireless Communications, vol. 17, no. 4, pp. 2233-2246, 2018, doi: 10.1109/TWC.2018.2790401.

[10] Y. Zeng and R. Zhang, "Energy-efficient UAV communication with trajectory optimization," IEEE Transactions on Wireless Communications, vol. 16, no. 6, pp. 3747-3760, 2017, doi: 10.1109/TWC.2017.2688328.

[11] V. V. Chetlur and H. S. Dhillon, "Downlink coverage analysis for a finite 3-D wireless network of unmanned aerial vehicles," IEEE Transactions on Communications, vol. 65, no. 10, pp. 4543-4558, 2017, doi: 10.1109/TCOMM.2017.2722500.

[12] Q. Wu, L. Liu, and R. Zhang, "Fundamental Trade-offs in Communication and Trajectory Design for UAVEnabled Wireless Network," IEEE Wireless Communications, vol. 26, no. 1, pp. 36-44, 2019, doi: 10.1109/MWC.2018.1800221.

[13] H. Ghazzai, M. B. Ghorbel, A. Kadri, M. J. Hossain, and H. Menouar, "Energy-efficient management of unmanned aerial vehicles for underlay cognitive radio systems," IEEE Transactions on Green Communications and Networking, vol. 1, no. 4, pp. 434-443, Dec. 2017, doi: 10.1109/TGCN.2017.2750721.

[14] Y. Zhou, N. Cheng, N. Lu, and X. S. Shen, "Multi-UAV-aided networks: Aerial-ground cooperative vehicular networking architecture," IEEE Vehicular Technology Magazine, vol. 10, no. 4, pp. 36-44, 2015, doi: 10.1109/MVT.2015.2481560.

[15] Q. Wu, Y. Zeng, and R. Zhang, "Joint trajectory and communication design for multi-UAV enabled wireless networks," IEEE Transactions on Wireless Communications, vol. 17, no. 3, pp. 2109-2121, 2018, doi: 10.1109/TWC.2017.2789293.

[16] H. Wang, J. Wang, G. Ding, L. Wang, T. A. Tsiftsis, and P. K. Sharma, "Resource allocation for energy harvestingpowered D2D communication underlaying UAV-assisted networks," IEEE Transactions on Green Communications and Networking, vol. 2, no. 1, pp. 14-24, 2018, doi: 10.1109/TGCN.2017.2767203.

[17] Q. Wu and R. Zhang, "Common throughput maximization in UAV enabled OFDMA systems with delay consideration," IEEE Transactions on Communications, vol. 12, no. 66, pp. 6614-6627, 2018, doi: 10.1109/TCOMM.2018.2865922.

[18] J. Lyu, Y. Zeng, and R. Zhang, "UAV-Aided offloading for cellular hotspot," IEEE Transactions on Wireless Communications, vol. 17, no. 6, pp. 3988-4001, 2018, doi: 10.1109/TWC.2018.2818734.

[19] A. Al-Hourani, S. Kandeepan and S. Lardner, "Optimal LAP Altitude for Maximum Coverage," IEEE Wireless Communications Letters, vol. 3, no. 6, pp. 569-572, 2014, doi: 10.1109/LWC.2014.2342736.

[20] N. T. Van, T. N. Do, V. N. Q. Bao and B. An, "Performance Analysis of Wireless Energy Harvesting Multihop Cluster-Based Networks Over Nakagami-m Fading Channels," IEEE Access, vol. 6, pp. 3068-3084, 2018, doi: 10.1109/ACCESS.2017.2787055.

[21] H. Li, J. Xu, R. Zhang, and S. Cui, "A general utility optimization framework for energy-harvesting-based wireless communications," IEEE Communications Magazine, vol. 53, no. 4, pp. 79-85, 2015, doi: 10.1109/MCOM.2015.7081079.

[22] N. T. Tung, P. M. Nam, P. T. Tin, "Performance evaluation of two-way with energy harvesting and hardware noises," Digital Communications and Networks, vol. 7, no. 1, pp. 45-54, 2020, doi: 10.1016/j.dcan.2020.04.003.

[23] P. T. Tin, D-V Phan, D-H Ha, T. N. Nguyen, M. Tran, and M. Voznak, "Non-Linear Energy Harvesting Based Power Splitting Relaying in Full-Duplex AF and DF Relaying Network: System Performance Analysis," Proceedings of the Estonian Academy of Sciences, vol. 69, no. 4, pp. 368-381, 2020, doi: 10.3176/proc.2020.4.06.

[24] A. A. Nasir, X. Zhou, S. Durrani and R. A. Kennedy, "Relaying Protocols for Wireless Energy Harvesting and Information Processing," IEEE Transactions on Wireless Communications, vol. 12, no. 7, pp. 3622-3636, 2013, doi: 10.1109/TWC.2013.062413.122042.

[25] A. Jeffrey and D. Zwillinger, "Table of integrals, series, and products," Academic press, 2007.

[26] T. Do, V. Nguyen, O. Shin and B. An, "Simultaneous Uplink and Downlink Transmissions for Wireless Powered Communication Networks," IEEE Communications Letters, vol. 23, no. 2, pp. 374-377, 2019, doi: 10.1109/LCOMM.2018.2885303. 